\title{
Digitale Plattformen als Geschäftsmodell
}

\author{
Daniel Moser, Christoph H. Wecht, Oliver Gassmann
}

Immer mehr Unternehmen sind Teil einer jungen, digital geprägten Firmengeneration. Angespornt von den Vorbildern im Silicon Valley revolutionieren sie mit innovativen Technologien und ausgefeilten Geschäftsmodellen gesamte Industrien. Während Google und Co. bereits zu den etablierten Konzernen zählen, arbeiten sich Startups wie der Lieferservice Delivery Hero, die Onlinebank N26, der Mobilitätsanbieter Limebike oder der Carsharingprovider Turo mit ähnlichen Strategien an die Spitze ihrer Industrien.

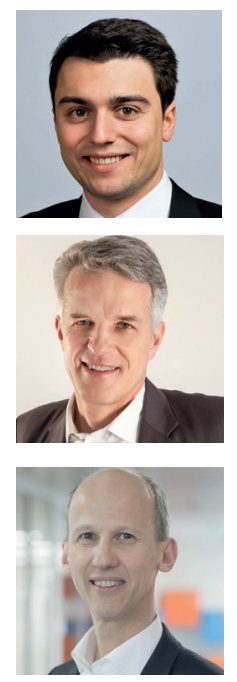

Dr. Daniel Moser ist Consultant bei Accenture. 2018 promovierte er am Institut für Technologiemanagement der Universität St. Gallen in Business Innovation, wo er davor auch Betriebswirtschaftslehre studiert hatte.

Prof. Dr. Christoph H. Wecht ist seit September 2017 Studiengangsleiter des Bachelorstudiengangs Management by Design an der New Design University (NDU) in St. Pölten, wo er zum Professor für Management berufen wurde.

Prof. Dr. Oliver Gassmann ist seit 2002 Professor für Technologie- und Innovationsmanagement an der Universität St. Gallen und Direktionsvorsitzender des dortigen Instituts für Technologiemanagement.
ए rmöglicht wird diese Entwicklung durch drei wesentliche Treiber:

1. eine zunehmende Digitalisierungvon Produkten, Prozessen und Dienstleistungen,

1. das Emporkommen von Plattformen,

2. die beschleunigte Innovation von Geschäftsmodellen.

Sensoren und die wachsende Vernetzung von Produkten, Dienstleistungen und Prozessen tragen einen wesentlichen Teil zur Digitalisierung bei. Daraus resultieren einerseits neue Funktionsumfänge von Produkten und fortschrittliche Dienstleistungen, andererseits die komplette Virtualisierung von Lieferketten. In einer zunehmenden Zahl der Fälle sind diese digitalisierten Produkte, Prozesse und Dienstleistungen mit Plattformen verbunden,

\section{In diesem Beitrag lesen Sie:}

- wie Plattformen entstehen,

- welches die Treiber hinter den Plattformen sind,

- wie Plattformen erfolgreich umgesetzt werden. welche die durch die Digitalisierung erzeugten Daten sammeln, speichern und auswerten. Basierend auf diesen Daten ermöglichen sich komplett neue Geschäftsmodelle, durch die Kundenwünsche immer besser adressiert und umgesetzt werden können. Diese oftmals digitalen Geschäftsmodelle fördern ihrerseits die wachsende Digitalisierung und bringen neue Generationen von Plattformen hervor.

Als zentrales Bindeglied zwischen digitalen Daten und innovativen Geschäftsmodellen haben sich Plattformen ihren festen Platz erkämpft. So wird heute die größte Zahl der Leistungen im Internet über Plattformen an den Kunden herangetragen. Diese sind aber noch viel mehr als nur ein digitaler Betriebskanal. In der Regel verbinden Plattformen in Kombination mit innovativen Geschäftsmodellen bis zu hunderte Millionen von Menschen. Plattformen ermöglichen außerdem den Austausch von Informationen oder wirtschaftlichen Leistungen und produzieren dabei eine enorme Menge von Daten. Menschen und Firmen, die mit der Plattform interagieren, bilden ein Ökosystem, ähnlich dem Vorbild aus der Natur. Durch den konstanten Austausch und die Interaktionen zwischen den Akteuren entwickelt sich das Ökosystem, aber auch die Plattform, weiter. Googles Android-Plattformnutzer generieren beispielweise eine Unmenge von Daten durch den Gebrauch ihrer Smartphones. Die erzeugten Daten verschwinden aber nicht im "Nirgendwo", sondern werden gezielt über die Plattform gesammelt und ausgewertet. Google nutzt die Informationen, die aus den Daten gewonnen werden. Einerseits wird damit die Plattform verbessert, andererseits werden sie unabhängigen Entwicklern zur Verfügung gestellt, die dadurch fortschrittlichere Applikationen für Android programmieren können. Die neuen Möglichkeiten, die sich durch die Nutzung der Plattform mit diesen Anwendungen ergeben, locken neue Plattformnutzer an und tragen somit zum Wachstum und zur Weiterentwicklung der Plattform und deren Ökosystems bei.

Was in einer zumeist digitalen, virtuellen Welt seinen Ursprung genommen hat, findet sukzessive den Weg in traditionelle Industrien, wo physische Produkte vernetzt und zu Platt- 

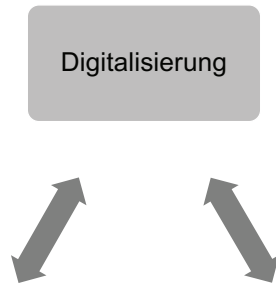

Plattformen

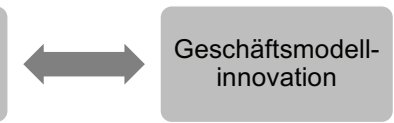

Bild 1: Drei Treiber zur (R)evolution von Industrien.

formen ausgebaut werden. Der deutsche Maschinenhersteller Trumpf hat mit Axoom ein Tochterunternehmen gegründet, welches die Vernetzung von Industrieanlagen mit einer proprietären loT-Plattform vorantreibt. Die Plattform ist dabei modular aufgebaut und verfügt über offene Schnittstellen, sodass die gesamte Produktionsanlage und nicht nur Maschinen von einzelnen, ausgewählten Herstellern eingebunden werden können. Einmal installiert, informiert die Plattform über den Zustand der Anlage und kann über "predicitve maintenance" längere und überraschend auftretende Produktionsausfälle verhindern. Daneben können die Leistung und Produktion der einzelnen Maschinen genau beobachten und somit Produktionsketten, bis hin zu den Lieferanten und Abnehmern, optimiert werden. Neben Axoom versuchen auch unterschiedliche andere Firmen die nächste führende Plattform für produzierende Unternehmen zu entwickeln. So investiert beispielsweise Siemens in die firmeneigene Mindsphere-Plattform, General Electric tritt mit Predix an den Markt oder die deutsche Kampf-Gruppe, welche weltweit führendim Bereich der Schneid- und Wickeltechnik für Folien ist, versucht seit 2017 mit der Plattform the@vanced eine Revolution in der Industrie herbeizuführen. Nachdem die großen Silicon Valley-Firmen mit ihren Plattformen um die Gunst der Endkonsumenten kämpfen, scheint sich in einem nächsten Schritt ein "Platform-War" um die Industrie zu entfachen. Neben intelligenten Produktionsmaschinen spielen auch vernetze Traktoren und Landmaschinen oder Datenplattformen in der Pharmaforschung eine immer wichtigere Rolle. Plattformen im industriellen Kontext stecken heute aber noch in den Kinderschuhen und Lösungen, welche eine oder mehrere Industrien dominieren, haben sich noch nicht abgezeichnet. Hinzu kommt, dass die Spielregeln für ein erfolgreiches Plattformmanagement für den B2B-Sektor teilweise neu geschrieben werden müssen, da die Skaleneffekte nicht mit denen von Plattformen im B2C-Bereich mit ihren vielen Nutzern verglichen werden können.

Neben der Wirtschaft nehmen Plattformen auch eine immer größere Rolle auf der politischen Bühne ein. Für das deutsche Bundeswirtschaftsministerium (BMWi) tragen Plattformen einen wichtigen Teil zu Digitalisierung des Landes bei und müssen in der digitalen Ordnungspolitik dementsprechend beachtet werden. Daneben standen Plattformen bereits mehrmals auf der Agenda des World Economic Forums, wo führende Köpfe einmal jährlich über die größten politischen, wirtschaftlichen und gesellschaftlichen Herausforderungen diskutieren. Schlussendlich adressiert die Europäische Union digitale Geschäftsmodelle und Plattformen aus unterschiedlichen Blickwinkeln, wie dem Datenschutz, der Verhinderung von Verbreitung von illegalen Inhalten oder der Transparenz von Algorithmen bezüglich deren Sortierung, Aufbereitung und Verbreitung von Informationen.

Fest steht, dass Wertschöpfung zunehmend über digitale oder semi-digitale Geschäftsmodelle betrieben wird. Plattformen bieten dafür die nötigen Strukturen und bilden die Infrastruktur, um solche Geschäftsmodelle umzusetzen. Dass diese die Grenzen zwischen einer rein digitalen und der physischen Welt verschwinden lassen, zeigt sich in verschiedenen Industrien. Das Rennen um die besten Plattformen hat längst begonnen. Führende Firmen von morgen können auf eine lange
Industrieerfahrung zurückgreifen und dieses Wissen mit digitalen Plattformen verbinden. Damit verändern und revolutionieren sie ganze Märkte. Spannend wird sein, wie sich die heutigen Industrie-Leader positionieren und die Herausforderungen der Digitalisierung und des Plattformbusiness meistern. Ein erster Schritt dazu ist ein breiteres Verständnis von Plattformen, wo sie herkommen und auf welche Komponenten man bei deren Aufbau und Management besonders zu achten hat.

\section{Geschäftsmodell als Motor der Plattform}

Um trotz der asymmetrischen Ressourcenverteilung eine erfolgreiche Plattform betreiben zu können, braucht sie ein passendes Geschäftsmodell. Externe Anbieter von Programmen und Dienstleistungen werden sich ohne die richtigen Anreize nicht an der Plattform und dem dazugehörenden Ökosystem mit kreativen Ideen beteiligen. Ohne ansprechende Inhalte bleiben jedoch die Nutzer aus und die Plattform liegt brach. Konsequenterweise müssen beide Gruppen entsprechend adressiert und incentiviert werden. Diese Situation ist vom Two-Sided-Market-Geschäftsmodell bekannt. Dieses beschreibt, wie zwei unabhängige Nutzergruppen mit oft nicht gleichgerichteten Interessen auf einer Plattform zusammengeführt werden. Die Kunst einer erfolgreichen Plattformstrategie liegt darin, zwischen diesen Nutzern sogenannte indirekte Netzwerkeffekte auszulösen. Das heißt, je mehr Nutzer von einer Gruppe auf der Plattform sind, desto attraktiver wird diese Plattform für die andere Usergruppe.

In der Praxis zeigt sich, dass viele Plattformbetreiber sich dazu motiviert sehen, eine breite Nutzerbasis aufzubauen, um über dritte Parteien indirekte Netzwerkeffekte zu initiieren. Spotify, die schwedische Musikstreaming-Plattform, unterhält beispielweise Lizenzverträge mit den führenden Plattenfirmen, um den Usern ein breites Musikangebot zur Verfügung zu stellen. Gleichzeitig kann die Musik mit einem 
Benutzeraccount kostenlos konsumiert werden. Das bringt Spotify bis dato monatlich 170 Millionen Nutzer. Um die Plattform zu monetarisieren, schaltet Spotify Werbung zwischen den Liedern. Werbeagenturen sehen den Vorteil, viele Millionen von Nutzern direkt adressieren zu können, weshalb sie zu Spotify kommen. Je mehr Nutzer sie so erreichen können, desto mehr Werbung werden sie veröffentlichen. Neben diesem indirekten Netzwerkeffekt hat es Spotify auch geschafft, Netzwerkeffekte mit Künstlern zu kreieren. So können diese über Spotify ihre Musik oder Podcasts veröffentlichen und haben so schnell ein Millionenpublikum für ihre Werke. Diese exklusiven Inhalte, die oft nur auf Spotify gefunden werden können, überzeugen dann ihrerseits wieder neue User, einen Account zu eröffnen und verstärken somit weiterhin die indirekten Netzwerkeffekte.

Oft wird eine Plattform zusätzlich mit Lock-In-Effekten ausgestattet. Das soll verhindern, dass ein einfacher und unkomplizierter Wechsel zu einer anderen Plattform vollzogen werden kann. Denn das würde die Zyklen der sich selbst verstärkenden Netzwerkeffekte zum Erliegen bringen. Ein möglicher Lock-In-Effekt wird beispielweise durch die Schaffung von künstlichen Medienbrüchen erzielt. Applikationen, die auf einer Plattform funktionieren, sind mit der Konkurrenzplattform in quasi sämtlichen Fällen inkompatibel. Ein Wechsel würde also zwangsweise den Verlust der bereits in Komponenten und Anwendungen getätigten Investitionen bedeuten. So überlegt sich eine Firma, die mit der SAP Plattform arbeitet und dort sämtliche, teils sehr teure Applikationen verwaltet genau, ob sie z.B. zur Salesforce Plattform wechseln soll, da dies neben Umschulungen von Mitarbeitern auch den Kauf und die Neuinstallation von allen Anwendungen bedeuten würde. Das erhöht die Wechselkosten für die Nutzer enorm und sichert den Plattformbetreiber, vor allem bei angebotenen Gratisleistungen, gegen einen plötzlichen Wegfall von Nutzern ab.
Bei der Monetarisierung der Plattformen ergeben sich wiederum unterschiedliche Optionen. So können Entwickler, Innovatoren oder Komplementäre, die Inhalte für die Plattform zur Verfügung stellen, für den Marktzugang belangt werden. Das ist beispielsweise bei den meisten „App-Stores" der Fall, wo nicht selten bis zu $30 \%$ des Verkaufspreises von der Plattform einbehalten wird. Aber auch eBay verlangt von den Verkäufern, welche mit ihren Verkaufsartikeln den kompletten Inhalt der Plattform kreieren, eine Gebühr für jeden Verkauf als Gegenleistung für den Marktzugang. Andere Plattformbetreiber entscheiden sich dafür, den Zugang grundsätzlich kostenlos anzubieten, dafür aber Werbeeinblendungen zu schalten. Das ist bei vielen Social-Media-Plattformen wie Facebook, Instagram, Snapchat oder Youtube der Fall. Letztendlich können aber auch die normalen Nutzer einer Plattform zur Kasse gebeten werden. Meist geschieht das über ein Freemium Modell, bei dem ein Basisangebot kostenlos bleibt während für Premiumfunktionen eine Gebühr verlangt wird, wie bei Spotify, Linkedin, Amazon oder N26. Egal welche Option gezogen wird, wichtig ist, dass nicht durch eine übermäßige oder falsch gewählte Monetarisierung die Netzwerkeffekte abgewürgt werden. Daher setzen auch viele Plattformen zuerst auf Wachstum und starke Netzwerkeffekte bevor die Wirtschaftlichkeit in den Vordergrund rückt.

Bei der Betrachtung des Gesamtgeschäftsmodells einer Plattform ergeben sich für den Betreiber folgende Implika- tionen für die vier Gestaltungsphären „Nutzenversprechen“ (Was?), „Zielkunden" (Wer?), "Wertschöpfungskette" (Wie?) und "Ertragsmechanik" (Wert?). Beim Nutzenversprechen und der Zielkundenadressierung ist der Plattformbetreiber demnach wesentlich auf die externen Komplementoren angewiesen. Während die Plattform einen grundlegenden Nutzen stiftet und ein möglichst breites Publikum adressiert, sind es vor allem diese unabhängigen Innovatoren, die mit ihren spezifischen Lösungen ganz gezielt kleinere Nutzergruppen ansprechen und somit einen individuellen Mehrwert durch die Plattform generieren. Dagegen ist der Einfluss auf das Gesamtsystem durch den Plattformbetreiber bei der Ertragsmechanik und der Wertschöpfungskette ungleich grösser. Die Ertragsmechanik muss so ausgestaltet werden, dass die Komplementoren genug vom Kuchen abbekommen und die Entwicklung von Anwendungen und Komponenten für die Plattform finanziell attraktiv ist. Gleichzeitig muss der Plattformbetreiber genügend profitabel sein, um das Grundangebot und die Infrastruktur der Plattform am Leben zu erhalten und auszubauen.

\section{Plattformpflege}

Damit neue Nutzer dem Ökosystem beitreten, muss der Zugang zur Plattform jederzeit einfach gewährleistet werden. Das kann über eine intuitiv gestaltete Benutzeroberfläche erreicht werden. Apple und Google unternehmen große Bemühungen, das Design

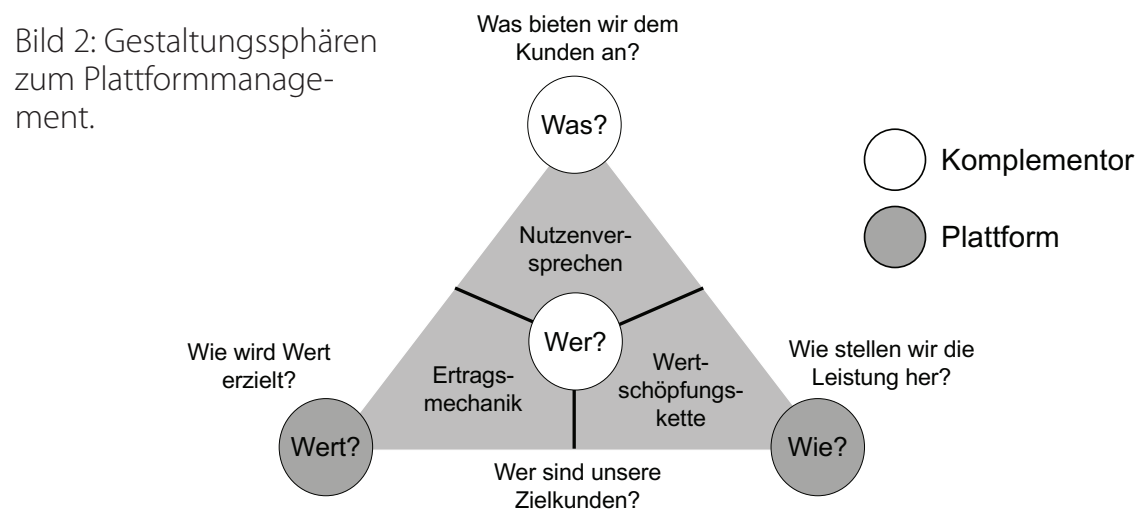




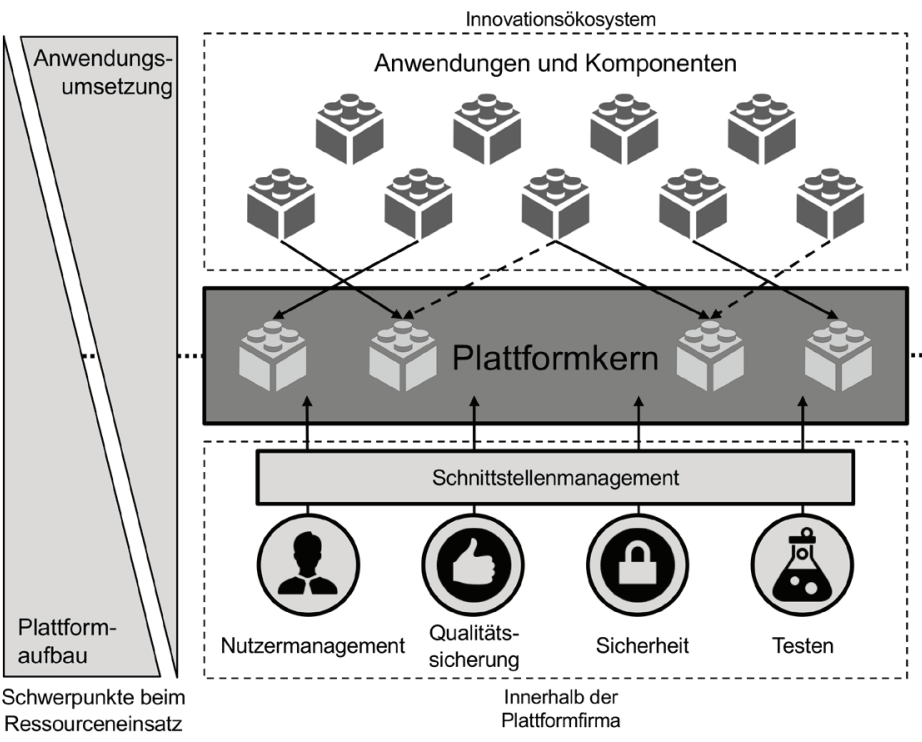

Bild 3: Schwerpunkte der Geschäftsmodellgestaltung für eine Plattform.

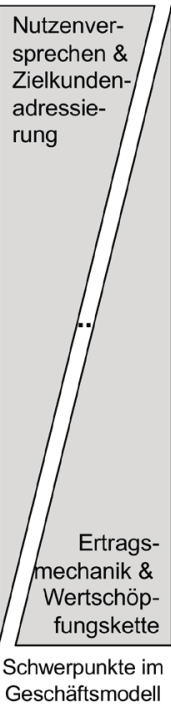

und die Bedienbarkeit ihrer Plattform laufend zu optimieren und zeitgemäßen Anforderungen anzupassen. Plattformfirmen greifen teilweise aber auch zu extremen Mitteln, um Nutzern den Zugang zu ihren Plattformen zu ermöglichen. So experimentiert Google mit Ballonen, die mit Antennen ausgestattet sind; Facebook mit Drohnen und Satelliten; Microsoft mit ungenutzten Fernsehfrequenzen, um Menschen in entlegenen Gebieten Zugang zum Internet und somit auch zu ihren Plattformen zu bieten. Gleichzeitig müssen aber auch die latenten Bedürfnisse, welche die Nutzer an die Plattform stellen, beobachtet, erkannt und interpretiert werden. Gewisse zentrale Funktionen, welche die Weiterentwicklung des Plattformkerns betreffen, müssen von der Plattformfirma initiiert werden und können somit nicht den externen Komplementoren überlassen werden. Apple und Google tun dies, indem dem Plattformkern neue Innovationen wie die Bezahlfunktion mit dem Handy über NFC (Near Field Communication) oder eine Assistenz wie Siri oder Google Assistant hinzugefügt werden.

Damit externe Entwickler aber überhaupt an der Plattform andocken können, müssen entsprechende Schnittstellen geschaffen und definiert werden. Diese sollten an der Stelle eingerichtet werden, wo die Ressourcenausstat- tung, um eine Frage oder ein Problem zu lösen, schwerpunktmäßig von der Plattformfirma zum externen Innovator übergeht. Bei den meisten Plattformen geschieht dies durch elektronische Verbindungen zu Software-Modulen. Dabei wird mit einer sogenannten API (Application Programming Interface) festgelegt, welche Dinge eine Applikation auf der Plattform ausführen kann. Gleichzeitig legt diese Programmierschnittstelle auch fest, welche Daten von der Plattform bezogen und welche Hardwarekomponenten angesteuert werden können. Somit werden die Rahmenbedingungen für die externen Innovationen, die mit der Plattform interagieren, festgelegt. Wird eine Funktion durch die API nicht erlaubt, findet sie auch keine Anwendung auf der Plattform. Facebook bietet beispielweise eine API zur Nutzung der grundlegenden Profilinformationen der User an. Will ein unabhängiger Entwickler Informationen wie Namen, E-Mail-Adresse, Freundeslisten und persönliche Präferenzen in seine Applikation einbinden, kann er auf die API der Facebook-Plattform zurückgreifen. Willigt der Nutzer der Weitergabe dieser Informationen zu, stehen diese dem Entwickler schnell und unkompliziert zur Verfügung. Die Verwendung von APIs kann aber auch weiter als über den bloßen Austausch von Daten gehen. Über die Android-Plattform wird beispielweise festgelegt, welche Hardwarekomponenten in einem Smartphone angesteuert werden können. Ohne die Kamera-API könnten unabhängige Entwickler nicht auf die Kamerafunktionen zugreifen und Applikationen wie Instagram wären nicht möglich, da damit keine Fotos gemacht werden könnten. Darüber hinaus sind viele Plattformanbieter dazu übergegangen, externen Entwicklern eine "Werkzeugkiste“ zur Verfügung zu stellen, um Applikationen zu programmieren, sogenannte Software Developerieren?

Dieser Beitrag basiert auf einem Buchkapitel des Buches «Digitale Transformation gestalten«, 2. Auflage, Hanser 2019 ISBN 978-3-446-45868-0

Schlüsselwörter:

Plattformmanagement, Geschäftsmodelle, zweiseitiger Markt

\section{Digital Platforms as Business}

Models

Value creation is increasingly driven by digital or semi-digital business models. Platforms offer the necessary backbone for such highly profitable business models. In many industries, these made the boundaries between a purely digital and a physical world disappear. Platforms offer the opportunity to realize enormous economies of scale and scope and thus enable companies to leverage their core business, experience and skills. At the same time, new business models can be developed and new customers acquired. Lock-ins are created by - often data-driven - stickiness of customers along their journey. This article shows how platforms are created, which drivers are behind this development and how platforms are successfully implemented.

Keyword:

digital platforms, business models, two sided market

\section{Kontakt:}

Prof. Dr. Oliver Gassmann Institut für Technologiemanagement Universität St.Gallen $\mathrm{CH}-9008$ St. Gallen E-Mail: oliver.gassmann@unisg.ch 